\title{
Die Uhr tickt etwas langsamer: erst Ende 2023 endet nun die Übergangsregelung für Rettungsassistenten*
}

Rico Kuhnke

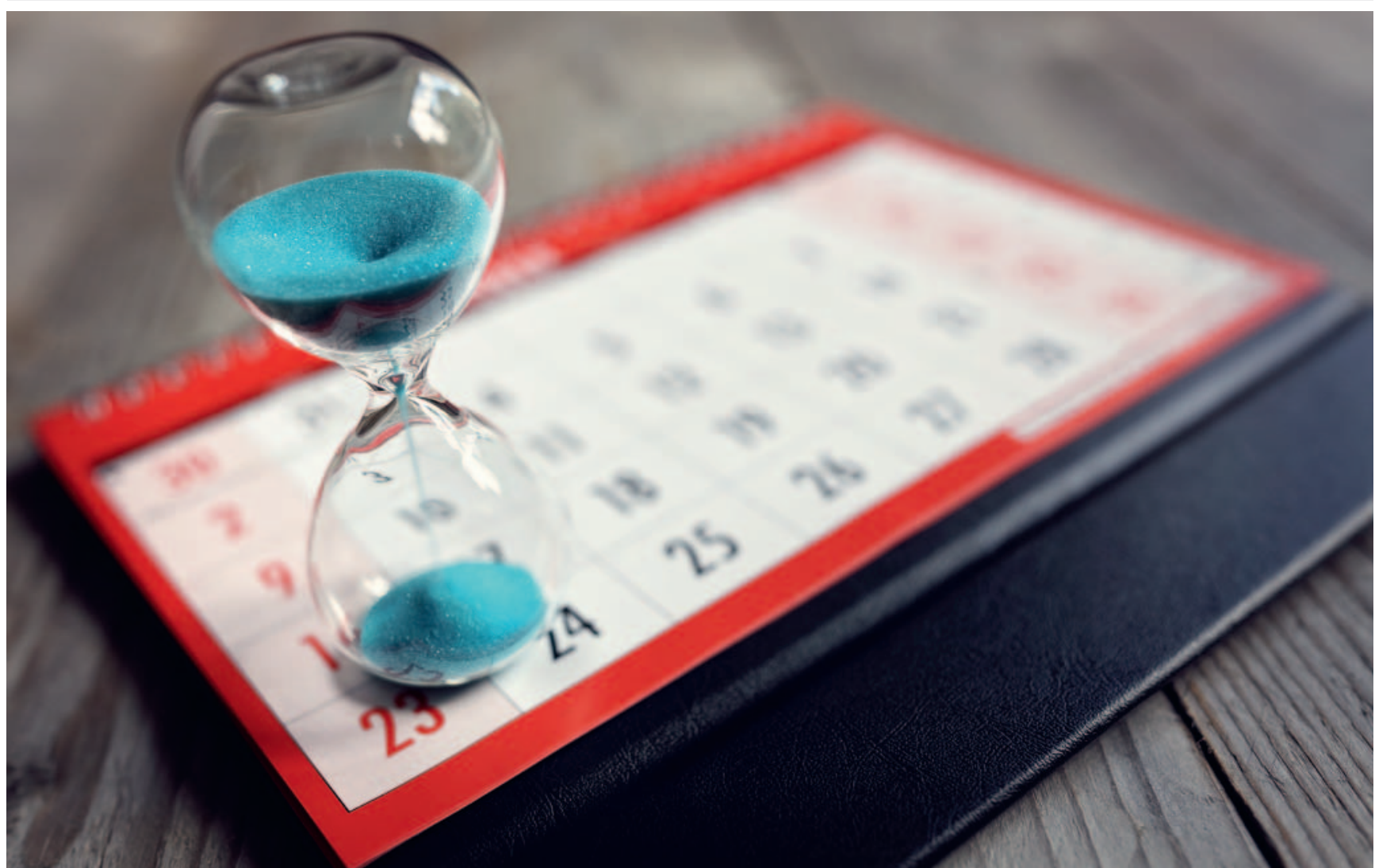

Quelle: Brian Jackson/stock.adobe.com

Am 29. November 2019 hat der Bundesrat einer Änderung des Notfallsanitätergesetzes zugestimmt. Nicht sieben sondern erst zehn Jahre nach Inkrafttreten des Notfallsanitätergesetzes (NotSanG) und seiner ergänzenden Ausbildungs- und Prüfungsordnung (NotSanAPrV) endet nun die Übergangsvorschrift nach § 32 NotSanG für Rettungsassistentinnen und Rettungsassistenten. Die Uhr tickt zwar, doch Rettungsassistenten bleibt nun noch bis Ende 2023 Zeit, an einer Ergänzungsprüfung oder alternativ an einer staatlichen Vollprüfung teilzunehmen.

\section{Übergangsvorschrift}

In der Übergangsvorschrift im §32 NotSanG werden die Voraussetzungen beschrieben, die es Rettungsassistentinnen und Rettungsassistenten ermöglichen, an einer staatli-

Dieser Beitrag ist eine aktualisierte Fassung der Druckversion „Die Uhr tickt: Ende 2020 endet die Übergangsregelung für Rettungsassistenten“. chen Prüfung teilzunehmen, um danach die Berufsbezeichnung Notfallsanitäterin/Notfallsanitäter führen zu dürfen.

\section{Merke}

Abhängig von der praktischen Tätigkeit als Rettungsassistent wird vor der Zulassung zu einer staatlichen Ergänzungsprüfung eine weitere Ausbildung von 480 Stunden bzw. 960 Stunden gefordert. 
Übergangsvorschriften für Rettungsassistenten

$\S 32$ NotSanG bis Ende 2023

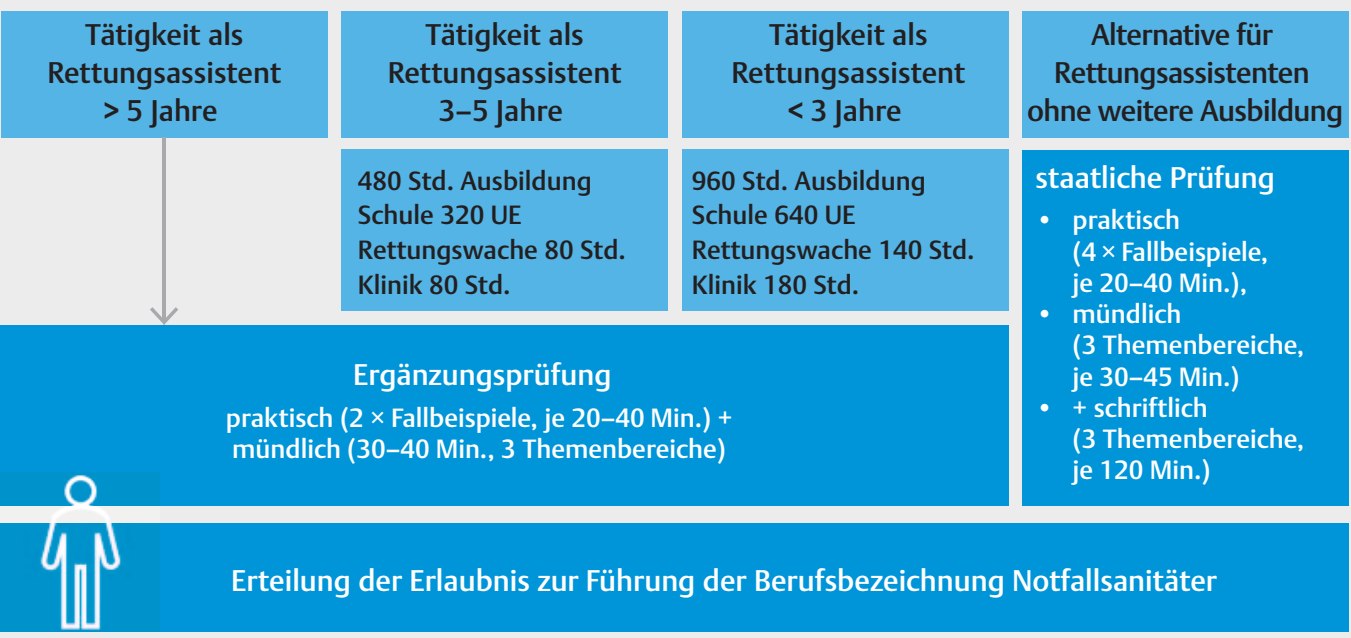

- Abb. 1 Übergangsvorschriften nach §32 NotSanG zur Erlangung der Berufsbezeichnung Notfallsanitäter.

Alternativ gibt es für Rettungsassistenten unabhängig von der beruflichen Praxis die Möglichkeit, sich direkt zu einer staatlichen Vollprüfung anzumelden. Entgegen der sonst üblichen Besitzstandregelungen, die eine Anerkennung von Berufen nach neuem Recht ohne weitere Auflagen ermöglichen, wollte der Gesetzgeber dies beim Notfallsanitäter explizit nicht. In der Begründung verweist der Gesetzgeber darauf, dass die nach neuem Recht ausgebildeten Notfallsanitäter im Vergleich zur bisherigen Ausbildung über erweiterte als auch vertiefte Kompetenzen verfügen müssen ( $\triangleright$ Abb. 1).

\section{Fristverlängerung}

Immer wieder wurde in den vergangenen Jahren auf eine Fristverlängerung spekuliert. Mit ihr verbunden war die Hoffnung vieler, dass der Gesetzgeber kurz vor Ablauf der Sieben-Jahre-Frist eine Verlängerung für Rettungsassistenten auf den Weg bringen würde. Ende Oktober 2019 haben die Regierungsparteien für viele überraschend eine Fristverlängerung ins Spiel gebracht. Im Antrag sollte die in $\S 32$ Absatz 2 NotSanG „sieben“ Jahre Übergangszeit durch „zehn“ Jahre ersetzt werden. Aufgrund einer Kopplung des Vorschlages zusammen mit einer Anpassungen der erweiterten Kompetenzen für Notfallsanitäter wurde der Vorschlag bereits einen Tag nach der Bekanntgabe zurückgezogen. Anfang November 2019 wurde die Fristverlängerung nun doch noch auf den Weg gebracht. Gemeinsam mit dem Gesetz über die Ausbildung zum Anästhesietechnischen Assistenten passierte der Vorschlag am 09. November 2019 den Deutschen Bundestag und am 29. November 2019 hat der Deutsche Bundestag der Änderung auf „zehn“ Jahre zugestimmt.

Mit der Änderung bleibt nun Rettungsassistenten drei Jahre mehr Zeit, die Ergänzungsprüfung oder alternativ eine Vollprüfung zum Notfallsanitäter zu absolvieren. Ungeklärt bleibt lediglich die Frage wie sich die Länder bei der Besetzung von Rettungsmitteln verhalten werden.

\section{Besetzung der Rettungsmittel}

Die Besetzung der Rettungsmittel ist in den Ländern nicht einheitlich. Sie wird in den jeweiligen Rettungsdienstgesetzen (RDG) näher geregelt ( $>$ Tab. 1 ).

In manchen Bundesländern wird das neu geschaffene Berufsbild des Notfallsanitäters noch immer völlig ignoriert (Bremen, Sachsen, Saarland), und es wird nach wie vor nur von Rettungsassistenten in der Notfallrettung gesprochen. Es bleibt abzuwarten, ob die Länder auf den Ablauf der Übergangsfrist warten und dann Anpassungen vornehmen. Bis zu einer Anpassung können Rettungsassistenten wie gewohnt eingesetzt werden. Einige Bundesländer haben bereits Übergangsregelungen in den RDG festgelegt (Niedersachsen, Brandenburg, Nordrhein-Westfallen, Hessen, Baden-Württemberg). Während in Hessen bereits seit 2018 keine Rettungsassistenten mehr als verantwortliche Beifahrer eingesetzt werden können, ist ihr Einsatz in Nordrhein-Westfallen bis 2026 vorgesehen. In Baden-Württemberg gilt die Übergangszeit bis 2020 und in Einzelfällen können Rettungsassisten- 
> Tab. 1 Besetzung der Rettungsmittel, Länderübersicht (Stand Oktober 2019).

Schleswig-Holstein

(n)

Mecklenburg-Vorpommern

Niedersachsen

Brandenburg

Berlin

Sachsen-Anhalt

\section{$\S 15$ SHRDG}

(2) RTW und Mehrzweckfahrzeuge sind mit zwei Personen zu besetzen, von denen eine Notfallsanitäterin oder Notfallsanitäter und die andere mindestens Rettungssanitäterin oder Rettungssanitäter mit Einsatzerfahrung ( $\$ 2$ Absatz 7) ist. Anstelle der Rettungssanitäterin oder des Rettungssanitäters kann auch eine Auszubildende oder ein Auszubildender zur Notfallsanitäterin oder zum Notfallsanitäter eingesetzt werden, die oder der die ersten 18 Monate der Ausbildung in Vollzeitform bereits absolviert hat; bei Ausbildung in Teilzeit verlängert sich der Zeitraum dergestalt, dass die entsprechenden Ausbildungsinhalte der achtzehnmonatigen Ausbildung in Vollzeitform absolviert sein müssen.

\section{§ 4 RDG M-V}

(2) Krankenkraftwagen, die in der Notfallrettung eingesetzt werden (Rettungswagen), müssen im Einsatz mit zwei Personen besetzt sein, von denen mindestens eine die Erlaubnis nach $\S 1$ des Rettungsassistentengesetzes oder eine Erlaubnis nach $\S 1$ des Notfallsanitätergesetzes besitzt. Als zweite Person kann auch eingesetzt werden, wer eine Ausbildung zur Rettungssanitäterin oder zum Rettungssanitäter erfolgreich abgeschlossen hat oder sich in der Ausbildung zur Notfallsanitäterin oder zum Notfallsanitäter befindet und über einen gleichwertigen Bildungsstand verfügt.

\section{$\S 21 \mathrm{HmbRDG}$}

(2) Bei der Notfallrettung sind Krankenkraftwagen im Einsatz mit mindestens einem Rettungssanitäter und einem Notfallsanitäter als Betreuer des Patienten zu besetzen.

\section{$\S 10$ NRettDG}

(2) Krankenkraftwagen sind im Einsatz in der Regel mit mindestens zwei Personen zu besetzen. Bei einer Notfallrettung ist im Rettungswagen in der Regel mindestens eine Person einzusetzen, die zum Führen der Berufsbezeichnung „Notfallsanitäterin“ oder „Notfallsanitäter“ berechtigt ist. Bis zum 31. Dezember 2022 kann anstelle einer Person nach Satz 2 eine Person eingesetzt werden, die zum Führen der Berufsbezeichnung „Rettungsassistentin“ oder „Rettungsassistent" berechtigt ist.

\section{$\S 30$ BremHilfC}

(4) In der Notfallversorgung sind Krankenkraftwagen im Einsatz mit mindestens zwei fachlich geeigneten Personen zu besetzen, von denen eine Person (Transportführer) Rettungsassistent nach dem Rettungsassistentengesetz und die andere mindestens Rettungssanitäter ist.

\section{$\S 6$ LRDPV}

(3) Rettungswagen sind mit mindestens zwei fachlich geeigneten Personen zu besetzen. Mindestens eine dieser Personen muss eine Ausbildung zur Notfallsanitäterin oder zum Notfallsanitäter erfolgreich abgeschlossen haben. Die zweite Person muss mindestens die Ausbildung zur Rettungssanitäterin oder zum Rettungssanitäter erfolgreich durchlaufen haben. Im Regelfall soll diese Person den Rettungswagen fahren.

Bis zum 31. Dezember 2020 können anstelle von Notfallsanitäterinnen und Notfallsanitätern auch Rettungsassistentinnen und Rettungsassistenten eingesetzt werden.

\section{$\S 9$ RDG Berling}

a) bei der Notfallrettung mit mindestens einer Notfallsanitäterin oder einem Notfallsanitäter im Sinne des Notfallsanitätergesetzes vom 22. Mai 2013 (BGBI. I S. 1348), das durch Artikel 30 des Gesetzes vom 18. April 2016 (BGBI. I S. 886) geändert worden ist, in der jeweils geltenden Fassung, sowie einer zum Führen des Krankenkraftwagens berechtigten Person, die über die Qualifikation Rettungssanitäterin oder Rettungssanitäter verfügt

\section{$\S 18$, RettDG LSA}

(1) Für die Notfallrettung und für die qualifizierte Patientenbeförderung eingesetzte Rettungstransportwagen, Intensivtransportwagen und Krankentransportwagen sind im Einsatz mit mindestens zwei Personen zu besetzen, von denen eine die Erlaubnis zur Führung der Berufsbezeichnung Notfallsanitäter nach dem Notfallsanitätergesetz oder eine gleichwertige Qualifikation besitzen muss, während die zweite Person die Ausbildung zum Rettungssanitäter abgeschlossen haben muss.

(2) Soweit in der Notfallrettung der Notarzt in einem gesonderten Rettungsmittel, insbesondere in einem Notarzteinsatzfahrzeug, zum Notfallort gebracht wird (Rendezvous-System), soll dieses mit einer Person, die die Ausbildung zum Notfallsanitäter abgeschlossen hat, besetzt werden.
Rettungsassistent wird im Gesetz nicht mehr erwähnt (seit 06.09.2019)

Eine Übergangszeit für Rettungsassistenten wird nicht genannt

Rettungsassistent wird im Gesetz nicht mehr erwähnt (seit 04.04.2017)

Übergangszeit für Rettungsassistenten bis Ende 2022

Notfallsanitäter wird im Gesetz nicht erwähnt

Übergangszeit für Rettungsassistenten bis Ende 2020

Rettungsassistent wird im Gesetz nur noch zum Einsatz auf NEF und ITW erwähnt

Rettungsassistent wird im Gesetz nicht mehr erwähnt (seit 06.05.2019) 
- Tab. 1 (Fortsetzung)

Nordrhein-Westfalen

\section{$\S 4$, RettG NRW}

(3) Für den Krankentransport ist mindestens eine Rettungssanitäterin oder ein Rettungssanitäter und für die Notfallrettung mindestens eine Rettungsassistentin oder ein Rettungsassistent beziehungsweise eine Notfallsanitäterin oder ein Notfallsanitäter zur Betreuung und Versorgung der Patientinnen und Patienten einzusetzen.

(7) Mit Ablauf des 31. Dezember 2026 wird die Funktion der Rettungsassistentin oder des Rettungsassistenten durch die Notfallsanitäterin oder den Notfallsanitäter ersetzt.

\section{$\S 12$, SächsRDG}

(2) Bei der Notfallrettung hat mindestens ein Rettungsassistent den Patienten zu betreuen, beim Krankentransport mindestens ein Rettungssanitäter im Sinne von $\S 8$ Abs. 2 des Rettungsassistentengesetzes. Von Satz 1 kann ausnahmsweise abgewichen werden, wenn anderenfalls das Fahrzeug nicht eingesetzt werden könnte.

\begin{tabular}{|c|c|}
\hline Hessen & $\begin{array}{l}\S 25, \text { HRDG-DV } \\
\text { (2) Auf Fahrzeugen für die Notfallversorgung darf der Leistungserbringer nur Personen } \\
\text { einsetzen, die } \\
\text { b) als Beifahrerin oder Beifahrer eine Erlaubnis nach } \S 1 \text { des Notfallsanitätergesetzes } \\
\text { besitzen und } \\
\text { 2. jährlich zu den Themenbereichen der Notfallversorgung fortgebildet werden. } \\
\text { Bis zum } 31 \text {. Dezember } 2017 \text { genügt die Besetzung mit einer Rettungsassistentin oder } \\
\text { einem Rettungsassistenten mit mehr als fünf Jahren Berufserfahrung. }\end{array}$ \\
\hline Thüringen & $\begin{array}{l}\S 16 \text {, ThürRettG } \\
\text { (2) Rettungsfahrzeuge sind im Einsatz mit mindestens zwei geeigneten Personen zu } \\
\text { besetzen. Dabei müssen die in der Notfallrettung eingesetzten Rettungsfahrzeuge mit } \\
\text { mindestens einem Rettungsassistenten oder Notfallsanitäter im Sinne des §14 Abs. } 4 \\
\text { Satz } 1 \text { besetzt sein. Das Landesverwaltungsamt kann auf Antrag des Notarztes für das } \\
\text { Notarzteinsatzfahrzeug hiervon Ausnahmen zulassen. }\end{array}$ \\
\hline Rheinland-Pfalz & $\begin{array}{l}\text { §22, RettDG } \\
\text { (3) Eine als Beifahrer eingesetzte Person ist dann fachlich geeignet, wenn sie beim } \\
\text { Notfalltransport mindestens eine Ausbildung als Rettungsassistent hat. }\end{array}$ \\
\hline Bayern & $\begin{array}{l}\text { Art. 43, BayRDG } \\
\text { (1) Krankenkraftwagen sind mit mindestens zwei geeigneten Personen zu besetzen. } \\
\text { Beim Krankentransport ist mindestens eine Rettungssanitäterin oder ein Rettungssani- } \\
\text { täter, bei der Notfallrettung ist mindestens eine Notfallsanitäterin oder ein Notfallsani- } \\
\text { täter zur Betreuung des Patienten einzusetzen. }\end{array}$ \\
\hline Baden-Württemberg & $\begin{array}{l}\text { §9, RDG } \\
\text { (1) Krankenkraftwagen und Notarzteinsatzfahrzeuge sind im Einsatz mit mindestens } \\
\text { zwei geeigneten Personen zu besetzen. 2Rettungswagen sind mit einem Rettungsassis- } \\
\text { tenten oder einem Notfallsanitäter zur Betreuung und Versorgung der Patienten zu } \\
\text { besetzen; als Fahrer und zweite Person fachlich geeignet ist, wer mindestens als } \\
\text { Rettungssanitäter ausgebildet worden ist. } \\
\text { (3) Der Einsatz von Rettungsassistenten nach Absatz } 1 \text { Satz } 2 \text { wird befristet bis zum } \\
\text { 31. Dezember } 2020 \text { zugelassen. Bei Vorliegen besonderer Gründe ist im Einzelfall die } \\
\text { Besetzung des Rettungswagens mit einem Rettungsassistenten bis spätestens zum } \\
\text { 31. Dezember } 2025 \text { zulässig. }\end{array}$ \\
\hline Saarland & $\begin{array}{l}\text { §4 SRettG } \\
\text { (1) Rettungsdienstfahrzeuge sind im Einsatz mit mindestens zwei fachlich und gesund- } \\
\text { heitlich geeigneten Personen zu besetzen. Bei der Notfallrettung hat mindestens ein } \\
\text { Rettungsassistent oder eine Rettungsassistentin, beim Krankentransport mindestens ein } \\
\text { Rettungssanitäter oder eine Rettungssanitäterin den Patienten oder die Patientin zu be- } \\
\text { treuen. Notarzt-Einsatzfahrzeuge sind neben dem Notarzt oder der Notärztin mit einem } \\
\text { Rettungsassistenten oder einer Rettungsassistentin zu besetzen. Rettungshubschrauber } \\
\text { müssen neben dem Piloten oder der Pilotin mit einem Rettungsassistenten oder einer } \\
\text { Rettungsassistentin, der oder die eine für den Einsatz auf Luftrettungsmitteln erforderliche } \\
\text { Zusatzausbildung abgelegt hat, und einem Notarzt oder einer Notärztin besetzt sein. }\end{array}$ \\
\hline
\end{tabular}

Übergangszeit für Rettungsassistenten bis Ende 2026

Notfallsanitäter wird im

Gesetz nicht erwähnt

Übergangszeit für Rettungsassistenten bis Ende 2017

Keine Begrenzung der Übergangszeit für Rettungsassistenten benannt

Notfallsanitäter wird im Gesetz nicht erwähnt

Rettungsassistent wird im Gesetz nicht mehr erwähnt (seit 01.05.2019)

Übergangszeit für Rettungsassistenten bis Ende 2020, in begründeten Einzelfällen bis Ende 2025

Notfallsanitäter wird im Gesetz nicht erwähn ten noch bis Ende 2025 auf einem Rettungswagen als verantwortlicher Beifahrer eingesetzt werden. In einigen Bundesländern wird keine Übergangszeit festgelegt
(Mecklenburg-Vorpommern, Thüringen). Es ist anzunehmen, dass das geltende RDG mit Ablauf der Übergangsfrist angepasst wird. 
Bei der Recherche sind besonders die Länder aufgefallen, in denen der Rettungsassistent bereits heute gar keine Erwähnung mehr findet (Schleswig-Holstein, Hamburg, Berlin mit Einschränkung, Bayern, Saarland). Interessant ist in diesem Zusammenhang, wie in den betroffenen Ländern zurzeit mit der Besetzung der Rettungsmittel umgegangen wird. Nach dem Gesetz dürften dort bereits heute Rettungsassistenten nur als Fahrer eingesetzt werden. Spannend bleibt nun die Frage ob die in den RDG der Länder geregelten Besetzungen der Rettungsmittel, aufgrund der Verlängerung der Übergangsfrist bis Ende 2023, für Rettungsassistenten angepasst werden.

\section{Merke}

Die Tendenz ist eindeutig und der Rettungsassistent als verantwortlicher Beifahrer ein auslaufendes Modell.

\section{Stichtagsregelung}

Zu Beginn hatte der Gesetzgeber die Berufserfahrung als Rettungsassistent zum 01.01.2014 als Berechnungszeitpunkt vorgesehen (,Stichtagsregelung“). Diese Regelung wurde am 04.04.2017 allerdings aufgehoben: Als Berufserfahrung gelten seitdem alle Zeiten, die bis zum Zeitpunkt des Antrags auf Zulassung zur Ergänzungsprüfung in der Tätigkeit als Rettungsassistent nachgewiesen werden können.

\section{Berufserfahrung als Rettungsassistent}

Im Kommentar zur Berufserfahrung weist der Gesetzgeber ausdrücklich darauf hin, dass nur dann von einer anrechenbaren Berufserfahrung ausgegangen werden kann, wenn diese ganz oder zu einem wesentlichen Teil zur Finanzierung des Lebensunterhalts gedient hat. Ehrenamtliche Tätigkeiten oder Teilzeitbeschäftigungen von weniger als $50 \%$ einer regulären Vollzeitbeschäftigung sind damit ausgeschlossen. Allerdings wird dies in den Bundesländern unterschiedlich gesehen.

\section{Alternative staatliche Prüfung}

Unabhängig davon gibt es für Rettungsassistenten die Alternative, eine staatliche Vollprüfung zu absolvieren. Diese ist zwar deutlich umfangreicher, dafür kann eine Anmeldung zur staatlichen Prüfung - zumindest theoretisch - ohne den Nachweis einer beruflichen Tätigkeit und ohne Vorbereitung erfolgen.

Dieser Weg ist nicht für alle Rettungsassistenten geeignet. Interessant ist er nur für Menschen, die im Lauf ihres Lebens die Berufsbezeichnung Rettungsassistent erworben und nicht oder nur wenig im Beruf gearbeitet haben, aber gern weiterhin ein berufliches Standbein im Rettungsdienst hätten - z. B. ein engagierter ehrenamtlicher
Kollege, eine Anästhesiepflegerin oder eine Medizinstudentin. Da die staatliche Prüfung deutlich umfangreicher ist als die Ergänzungsprüfung, sollten sich Interessierte auf eine intensive Vorbereitungszeit einstellen.

\section{Ticktack - die Uhr tickt trotzdem}

Gehören Sie zu den Rettungsassistenten, die derzeit noch überlegen, ob sie sich einer Prüfung stellen? Dann sollten Sie nicht mehr allzu lange warten. Zwar haben Sie mit der Verlängerung nun noch weitere drei Jahre Zeit, allerdings sollten Sie bei Ihren Überlegungen beachten, dass Ihr Einsatz als verantwortlicher Beifahrer auf einem Rettungsmittel, zumindest langfristig, nicht mehr gesichert ist.

\section{Rettungsassistenten sterben aus}

Der Gesetzgeber hat im $\S 30$ NotSanG geregelt, dass Rettungsassistenten ihre Berufsbezeichnung weiterführen dürfen. Offen bleibt dabei die Frage, in welcher Funktion Rettungsassistenten künftig im Rettungsdienst beschäftigt werden. Bereits heute ist die Durchführung erweiterter Maßnahmen (SOP, Algorithmen, Handlungsempfehlungen) in vielen Regionen Notfallsanitätern vorbehalten. In Berlin und Thüringen ist dies sogar im Rettungsdienstgesetz (RDG) verankert.

\section{Merke}

Es ist anzunehmen, dass Rettungsassistenten künftig bestenfalls als Fahrer in der Notfallrettung eingesetzt werden.

\section{Hindernis Arbeitgeber}

Rettungsassistenten, die eine Ergänzungsprüfung ablegen möchten, aber vom Arbeitgeber nicht angemeldet oder vertröstet werden, sollten unbedingt aktiv werden. Wenden Sie sich an den Betriebsrat und dokumentieren Sie Ihre Anstrengungen um eine Anmeldung zur Prüfung. Dies kann insbesondere für das weitere Beschäftigungsverhältnis und für Ihre Vergütung notwendig sein. Regelhaft werden Notfallsanitäter bereits heute besser bezahlt als Rettungsassistenten - unabhängig von der Tätigkeit, die sie im Rettungsdienst ausüben.

\section{Rettungsassistent bleiben}

Sicher gibt es Gründe, sich nicht mehr einer Prüfung zu stellen. Insbesondere betrifft dies vermutlich ältere Kollegen, die angesichts des bevorstehenden Ruhestands von einer Prüfung absehen. In jedem Fall sollten die Betroffenen im Vorfeld die länderrechtlichen Bestimmungen für die weitere Beschäftigung als Rettungsassistent prüfen. Auch ist es sinnvoll, vor einer endgültigen Entscheidung mit der Rentenversicherung den Beginn der Altersrente 
zu klären. Erst danach kann man für sich prüfen, ob und wie man die letzten Jahre im Rettungsdienst als Rettungsassistent verbringt.

Manch eine Rettungsassistentin oder ein Rettungsassistent spekuliert bei der Entscheidung, Rettungsassistent zu bleiben, auf eine Beschäftigung auf dem Notarzteinsatzfahrzeug (NEF). Diese Plätze sind aber begrenzt und es gibt bereits heute Länder, in denen auch für das NEF die Mindestqualifikation Notfallsanitäter vorgeschrieben ist.

\section{Arbeitsrechtliche Konsequenzen}

Auch hört man immer wieder von Kollegen, die sich einer Weiterqualifizierung zum Notfallsanitäter verweigern möchten. Darauf zu hoffen, dass die Arbeitgeber genügend Stellen für Rettungsassistenten vorhalten, ist allerdings sehr kurzsichtig. Spätestens dann, wenn die Rettungsassistenten nicht mehr regulär eingesetzt werden können, werden die Arbeitgeber prüfen, ob eine Beschäftigung der verbliebenen Rettungsassistenten als Rettungssanitäter erfolgen kann. Damit verbunden wäre selbstverständlich eine entsprechend geringere Vergütung.

\section{FAZIT}

Mittlerweile haben wahrscheinlich die allermeisten Rettungsassistenten ihre Prüfung zum Notfallsanitäter erfolgreich abgeschlossen. Nach anfänglichen Problemen haben sich die Vorbereitungsphase in den Schulen und die anschließende Prüfung eingespielt. Die Durchfallquoten liegen deutlich niedriger als noch zu Beginn; spätestens beim zweiten Anlauf wird die Wiederholungsprüfung in aller Regel bestanden. In Baden-Württemberg liegt die Quote der Menschen, die auch die Wiederholungsprüfung nicht bestehen, bei unter $1 \%$. Aus diesem Grund kann man den verbliebenen Rettungsassistenten nur Mut machen, sich einer Prüfung zum Notfallsanitäter zu stellen.
Die Qualität im Rettungsdienst hat sich durch die Vorbereitungslehrgänge und die intensive persönliche Vorbereitung der Einzelnen auf die praktische und mündliche Ergänzungsprüfung schon wahrnehmbar verbessert. Das Ziel des Gesetzgebers, die ehemaligen Rettungsassistenten mit den weiterführenden Kompetenzen und Schlüsselqualifikationen vertraut zu machen, konnte erreicht werden.

\section{Interessenkonflikt}

Die Autoren geben an, dass kein Interessenkonflikt besteht.

\section{Autorinnen/Autoren}

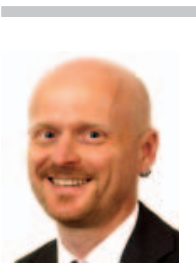

\section{Rico Kuhnke}

MA. Gesamtschulleiter der DRK-Landesschule Baden-Württemberg. Er war viele Jahre als Lehrrettungsassistent tätig und hat sein pädagogisches Masterstudium berufsbegleitend abgeschlossen. Er ist Notfallsanitäter und Mitherausgeber von retten!

\section{Korrespondenzadresse}

\section{Rico Kuhnke}

Schulleiter

Deutsches Rotes Kreuz

Landesschule Baden-Württemberg

Karl-Berner-Str. 6

72285 Pfalzgrafenweiler

r.kuhnke@drk-Is.de

\section{Bibliografie}

DOI https://doi.org/10.1055/a-0962-3580

retten 2020; 9: e1-e6

(c) Georg Thieme Verlag KG, Stuttgart · New York ISSN 2193-2387 